\title{
EFFECT OF SOME WEED CONTROL PACKAGES ON SEED COTTON YIELD AND FIBER PROPERTIES OF SOME COTTON GENOTYPES (GOSSYPIUM BARBADENSE, L.) AND ITS ASSOCIATED WEEDS
}

\author{
SOLIMAN, I. E. ${ }^{1}$, AZZA E. KHAFFAGY ${ }^{1}$, A. M. GHALWASH ${ }^{1}$ and \\ AMAL S. ABD EL-AAL ${ }^{2}$
}

1. Weed Research Central Laboratory, ARC, Giza, Egypt.

2. Cotton Res. Institute, ARC, Giza, Egypt.

(Manuscript received 26 November 2013)

\begin{abstract}
Two field experiments were conducted during 2011 and 2012 seasons at Sakha Agricultural Research Station, to investigate the performance of three cotton genotypes (Giza 86, Giza 88 and the new promising hybrid Giza $89 \times 86$ ) under some weed control packages (pendimethalin, butralin, fluazifop-p-butyl and sethoxydim) plus one hand hoeing for each herbicide in addition to the hand hoeing twice for controlling total weeds and their effects on some vegetative characters, yield components and fiber properties of cotton. Results indicated that the promising hybrid Giza $89 \times 86$ decreased dry weight of broad-leaved, grassy and perennial weeds by $27.6,31.9$ and $26.8 \%$ respectively, at second survey, also recorded the highest values of yield and its components, as compared to the genotype Giza 86 . followed by Giza 88 in both seasons. Moreover, all weed control packages significantly decreased weeds parameters and increased yield components in both seasons. Also, gave highly significant increase in seed cotton yield (Kantar /Fadden). The highest values were obtained with (pendimethalin, butralin) plus one hand hoeing treatments, followed by hand hoeing twice and (fluazifop-p-butyl, sethoxydim) plus one hand hoeing by $66.7,62.3,63.7$, 56.5 and $46.4 \%$ and increased micronaire reading by $34.0,34.0,25.2$, 30.5 and $28.0 \%$ respectively, as compared to the control treatment. The interaction between cotton genotypes and weed control packages exerted a significant integrated impact on weeds characters, and this reflected on increases in seed cotton yield components and fiber quality. From results of correlation analysis, the dry weight of total weeds biomass were negative correlated with cotton yield and micronaire reading. These results indicated that under heavily infested soil with weeds, it is better to grow the promising hybrid Giza $89 \times 86$ and Giza 88 with the application of weed control packages such as (pendimethalin or butralin) plus one hand hoeing or hand hoeing twice. These practices gave the highest reduction in weeds density and increased cotton yield and its components and improved yield cotton and fiber quality.
\end{abstract}

\section{INTRODUCTION}

Cotton (Gossypium barbadense, L.). is an important crop in Egypt for local consumption of fibers and oils and exportation due its high as long staple fiber. In 2013 the cultivated area with cotton arrived 520000 faddan (Anonymous 2102). Growth and yield of cotton is substantially reduced by weed competition. Bukun 
(2004), in Turkey found that weeds should be eliminated from 1-2 weeks up to 11-12 weeks of plant emergence and weed control strategies should be done in these periods by enhancing the herbicides use and other methods of weed management including cultivations. Cotton plants is a weak competitor for weeds due to its prolonged season especially in early growing periods. Ferrel et al. (2001) in India found that the infestation of weed flora in cotton crop reduced the yield by 1.28 and 1.6 ton /ha compared to 2.41 and 2.33 ton/ha from weed free cotton field, and the severity of weed competition depending on weed densities and their compositions. Several scientists have studied the influence of different weed species exist in cotton fields. In all cases, yield has been the most sensitive indicator of weed competition. (Echinochola crus-galli) can be a problem for irrigated cotton grown in the Western United States (Miller et al. 1961). Competition from 1 to 3_plants of (Cyperus esculentus) per three cotton plants reduced cotton dry weight, but yield was reduced most when cotton was stressed by low fertility or low soil moisture. Seed cotton yield in hand weeded control plots averaged $14 \%$ higher than in plots where the weed remained throughout the season (Keeley et al. 1973 and Keeley and Thullen 1975). Competition with (Cyperus esculentus) for 6 to 8 weeks reduced yield $20 \%$ and full season competition cut it $34 \%$. They also mentioned that, micronaire readings were lower for cotton samples collected from plots that contained nutsedge for longer than 4 week than from samples collceted from weed -free plots indicating delayed crop maturity, also growth and yield and delayed fiber maturity. (Xanthium pensylanicum) also is a very serious problem weed in cotton where at densities of 1 to 10 plants per 10 cotton plants reduced yield by more than $20 \%$ up to more than $80 \%$ (Buchanan and Burns, 1971).

Concerning the use of herbicides for weed control in cottons Several workers have shown that dinitroaniline herbicides e.g. pendimethalin were more effective in controlling summer weeds and need light hoeing as complements (Fayed et al. 1983, and Khan et al. 2001). They obtained highest seed cotton yield with application of pendimethalin. Moreover, Dilbaugh et al. (2009) indicated that application of pendimethalin $33 \%$ on dry bed furrow before applying irrigation produced $82.5 \%$ broad leaf and $84.1 \%$ narrow leaf control which ultimately led towards obtaining seed cotton yield of $2689 \mathrm{~kg}$ ha- 1 which was $115.1 \%$ higher than the weedy check. El-Maghraby (1971) indicated that hand hoeing increased the number of bolls/ plant, mean while, fiber strength, elongation percent and fiber fineness were not affected by using pre-emergence herbicides at different doses. Also Ghaly (1981) found that weed control treatments had a significant effect on seed cotton yield and fiber fineness. Lint percentage, fiber length and strength were 
insignificantly affected by weed control treatments. Nabil et al. (1983) stated that the application of Stomp before planting gave the highest lint percentage, micronaire value and oil percentage. El- Shaer et al. (1985) found that seed cotton yield per plant and per faddan as well as number of opened bolls per plant were increased. However, fiber length, strength and micronaire reading were not affected by weed control treatments. Hussain et al. (1989) compared the effects of 4 weed control treatments on cotton. They found that all treatments increased number of bolls per plant, seed cotton weight per plant and lint cotton yield. Abd El-Rehim et al. (1995) found that fiber length at $50 \%$ and $2.5 \%$ S.L., micronaire reading, fiber strength uniformity ratio and fiber elongation were not significantly affected by either hand hoeing or the various herbicidal treatments. However, fiber length uniformity ratio, fiber strength at both zero and 1/8 inch gauge length, fiber stiffness, fiber toughness and yarn strength were significantly increased by hand hoeing or different herbicidal treatments.

Ghourab (1990) stated that the herbicide combination Goal + Amex showed higher seed cotton yield per plant or faddan than single application of these herbicides. Micronaire reading was significantly affected by herbicidal treatments in one season only. He added that weed control treatment had insignificant effect on fiber strength, elongation and fiber length. Meanwhile, Abd El-Bary et al. (2010) mentioned that the cotton promising cross Giza $89 \times 86$ ) exceeded the commercial genotype Giza 86 by about $10 \%$ of cotton yield. For these reasons, the objective of this investigation was to determine the degree of integration between chemical and mechanical weed control and cotton genotypes on associated weeds, cotton yield and its fiber properties.

\section{MATERIALS AND METHODS}

Two field experiments were conducted at Sakha Agricultural Research Station Center, Kafer El-Sheikh Governorate during 2011 and 2012 seasons. The aim was to study the performance of three cotton genotypes (Gossypium barbadense, L.) , Giza 88, Giza 86 and the promising hybrid Giza 89 x Giza 86, under six weed control packages on their associated weeds and its prabroly and fiber properties. All genotypes were planted in April, $5^{\text {th }}$ in both seasons. Soil texture of the experimental site in both seasons was clay loam (Table 1 ). 
Table 1. Mechanical and chemical analysis for experimental soil.

\begin{tabular}{|c|c|c|}
\hline soil analysis & 2011 & 2012 \\
\hline Sand $\quad(\%)$ & 16.7 & 18.9 \\
\hline Silt $\quad(\%)$ & 33.14 & 32.73 \\
\hline Clay $\quad(\%)$ & 50.2 & 48.4 \\
\hline Soil textural class & Clay & Clay \\
\hline$(\mathrm{Ph})$ & 8.00 & 8.09 \\
\hline Soil salts E.C.(m.mhos/cm) & 2.49 & 3.01 \\
\hline Organic matter (\%) & 1.54 & 1.30 \\
\hline Available nitrogen (ppm) & 19.35 & 22.00 \\
\hline Available phosphorus (ppm) & 15.00 & 20.00 \\
\hline Available potasium (ppm) & 278.1 & 283.00 \\
\hline
\end{tabular}

Eighteen treatments of each experiment were arranged in a split plot design with four replicates. The sub plot area was $10.5 \mathrm{~m}^{2}$ contained five ridges $3.5 \mathrm{~m}$ length and $60 \mathrm{~cm}$ apart. The distance between hills was $25 \mathrm{~cm}$ apart. Seedlings were thinned to secure the required number of plants before the $2^{\text {nd }}$ irrigation. Cotton plants were picked on twice, the firstly picked on Oct. $5^{\text {th }}$ and secondly on Nov. $1^{\text {st }}$ in the two seasons. The treatments were as follow:

I. Main plots (cotton genotypes):-

1- Giza 86. 2-Giza $88 . \quad 3-T h e$ promising hybrid Giza 89 x 86).

II. Sub plots (integrated weed control packages):-

1. Amex (butralin, 48\% EC) 4-(1,1-dimethylethyl)-N-(1-methylpropyl)-2,6dinitrobenzenamine) at rate of $2.5 \mathrm{l} /$ fad., applied post sowing, followed by one hand hoeing after 45 days from sowing.

2. Stomp Extra (pendimethalin, 45.5\% CS) N-(1-ethylpropyl)-3,4-dimethyl-2,6dinitrobenzenamine at rate of $1.7 \mathrm{l} /$ fad., applied post sowing, followed by one hand hoeing after 45 days from sowing.

3. Fusilade Super (fluazifop-P- butyl $12.5 \%$ EC) butyl $®-2-[4-[[5-($ trifluoromethyl)2-pyridinyl]oxy] henoxy]propanoate at rate of $1.5 \mathrm{l} /$ fad., applied at 21 days after sowing followed by on hand hoeing after 45 days from sowing.

4. Nabu.S (sethoxydim, $12.5 \% \quad$ EC) (t)-(EZ)-2-(1-ethoxyminobutyl)-5-[2(ethylththio) propyl]-3-hydroxycylohex-2-enone) at the of $1.5 \mathrm{l} /$ fad., applied at 21 days after sowing followed by one hand hoeing after 45 days from sowing.

5. Hand hoeing (twice), at 30 and 45 days from sowing.

6. Control (untreated).

Herbicides in both experiments were sprayed by knapsack sprayer CP3 with water volume of 200 liters water/fad. All recommended agronomic practices of cotton were adopted throughout both growing seasons. The collected data were as follows:

\section{A. Dry weight of weeds $\left(\mathbf{g} / \mathbf{m}^{2}\right)$ :-}

Weeds were hand pulled at random from one square meter of each plot after 75 and 105 days from sowing and classified into four categories, broad leaf, grassy, 
perennial and total weeds. The dry weight was determined as $\left(\mathrm{g} / \mathrm{m}^{2}\right)$ after drying in a forced draft oven at $70 \mathrm{C}^{\circ}$ for 48 hours.

\section{B. Cotton vegetative characters and yield components:}

At harvest time, samples of ten plants were collected at random from the central ridges of each plot to assess the following criteria: plant length $(\mathrm{cm})$, number of fruiting branches / plant, number of bolls / plant, boll weight ( $\mathrm{g}$ ), seed index, lint and seed cotton yield was estimated as kentar per faddan. (Boll weight: the average weight of ten open bolls in grams, seed index: the weight of 100 seeds in grams, lint percentage: ratio of lint to seed cotton expressed as a percentage).

\section{Cotton fiber properties:}

The cotton fiber properties studied were: Micronaire reading, fiber length: the length at $2.5 \%$ span length were measured, uniformity ratio was calculated (Sundaram, 1980), pressely index as measured by Fibrograph (ASTM, D 1447-83, 1984), and fiber length ( $\mathrm{mm}$ ) the cotton fiber properties were estimated in Cotton Agronomy Department Cotton Res. Institute (ARC).

\section{Correlation study:}

Simple correlation matrix was carried out for the two seasons to investigate the relationship between dry weight of different weed categories and cotton genotypes yield and its components according to Steel and Torrie (1980).

\section{Statistical analysis:}

Results were analyzed as split plot design with four replicates and a combined statistical analysis for the two years following the procedure outlined by Snedecor and Cochran (1980) Data were exposed to Barttelt test and were homogenous, for this reason the combined data of the two years were presented in the following results. Means were compared according to Duncan's multiple range test (1955).

\section{RESULTS AND DISCUSSION}

\section{A: Effect on weeds}

The most dominant weeds in experimental fields were wild jute (Corchorus olitorius L.), cocklebur ( Xanthium brasilicum L.), white goosefoot ( Amaranthus album L.), common purslane (Portulaca olerceae L.), and black nightshade ( nigrum L.), as broad leaf weeds and deccan grass (Echionchloa colona L.), Panz (Dinebra retroflexa L.), and bermuda grass (Cynodon dactylon L.,) as grassy weeds and purple nutsedge (Cyperus rotundus L.) as perennial weeds in both 2011 and 2012 seasons. Table (2) show mean squares of variance for the effects of cotton genotypes, weed control packages and their interactions on dry weight of weeds were statistically significant at $1 \%$ level of probability. 
Table 2. Means squares from analysis of variance for the effect of cotton genotypes weeds control and their interaction on dry weight of weeds $\left(\mathrm{g} / \mathrm{m}^{2}\right)$ (combined data of 2011 and 2012).

\begin{tabular}{|c|c|c|c|c|c|c|c|c|c|}
\hline \multirow{4}{*}{ Source } & \multirow{4}{*}{ DF } & \multicolumn{8}{|c|}{ Days after sowing } \\
\hline & & \multicolumn{4}{|c|}{75} & \multicolumn{4}{|c|}{105} \\
\hline & & \multicolumn{8}{|c|}{ Dry weight of weeds in $\left(\mathrm{g} / \mathrm{m}^{2}\right)$} \\
\hline & & $\begin{array}{l}\text { Grasses } \\
\text { weeds }\end{array}$ & $\begin{array}{c}\text { Broadleaf } \\
\text { weeds }\end{array}$ & $\begin{array}{c}\text { perennial } \\
\text { weeds }\end{array}$ & $\begin{array}{c}\text { Total } \\
\text { Weeds }\end{array}$ & $\begin{array}{c}\text { Grasses } \\
\text { weeds }\end{array}$ & $\begin{array}{c}\text { Broadleaf } \\
\text { weeds }\end{array}$ & $\begin{array}{c}\text { Perennial } \\
\text { weeds }\end{array}$ & $\begin{array}{c}\text { Total } \\
\text { weeds }\end{array}$ \\
\hline Year $(Y)$ & 1 & $6611.34 * *$ & 1026.8 & 66.9 & 1688.23 & 389.12 & $2957981.5^{* *}$ & $118094.3^{* *}$ & 4339942.96** \\
\hline$R \times Y$ & 4 & $867.63 * *$ & $2838.5^{*}$ & 476.02 & 4376.04* & 5889.43 & 19150.1 & 94569 & 17722.73 \\
\hline Genotypes ( & & & & & & & & & \\
\hline G ) & 2 & $15978.86 * *$ & $49063.4 * *$ & $44247 * *$ & $307757.07^{* *}$ & $127125.48^{* *}$ & $1328060.46 * *$ & $60095.47 * *$ & $3057096.04 * *$ \\
\hline$Y \times G$ & 2 & $1599.62 * *$ & $10511.58^{* *}$ & $945.82 * *$ & $24604.29 * *$ & $24286.04 *$ & $44346.13^{*}$ & $24840.51^{* *}$ & $65682.7^{*}$ \\
\hline Error & 8 & 321.28 & 1640.95 & 502.64 & 2288.68 & 10428.7 & 28737.02 & 1239.27 & 38040.63 \\
\hline $\begin{array}{l}\text { Treatment } \\
\text { (T) }\end{array}$ & 5 & $36093.57 * *$ & $193917.37 * *$ & $64080.7^{* *}$ & $734629.81 * *$ & $470080.08^{* *}$ & $1777848.8^{* *}$ & $91757.78^{* *}$ & $5229997.8^{* *}$ \\
\hline$Y \times T$ & 5 & $1479.59 * *$ & $22531.75^{* *}$ & $1676.1 * *$ & $24722.48 * *$ & $18707.72^{* *}$ & $94960.44 * *$ & $30177.57 * *$ & $365795.64 * *$ \\
\hline$G \times T$ & 10 & $6264.25 * *$ & $11528.65^{* *}$ & $7822.04 * *$ & $72449.69 * *$ & $48064.48^{* *}$ & $175254.36 * *$ & $9051.53 * *$ & $511907.42 * *$ \\
\hline$Y \times G \times T$ & 10 & 121.17 & 1355.42 & 51.42 & 2275.57 & 860417* & 5539.4 & $4806.03 * *$ & 13947.47 \\
\hline Error & 60 & 619.49 & 1887.6 & 488.74 & 3327.7 & 8056 & 21073.53 & 646.04 & 24034.8 \\
\hline
\end{tabular}

Values followed by $*$ are significantly at $(p=0.05)$. 


\section{1- Effect of cotton genotypes:}

Results in Table (3) show that dry weight of weed groups was affected by cotton genotypes at 75 and 105 days from sowing. Giza 86 genotype plots had the heaviest dry weight of weeds while, Giza 88 had the moderate weights for grassy, broad-leaved, perennial and total weeds at 75 and 105 days from Sowing. The promising hybrid Giza 89 × 86 cotton genotype gave the broadest spectrum of weed control and decreased dry weight of weed groups by $27.6,31.9,18.7$ and $26.8 \%$ for weeds, respectively at 75 days after sowing as compared with Giza 86 where the respective values at 105 days were $17.46,27.25,18.6$ and 24\%, respectively. These results sustained that Giza $89 \times 86$ hybrid genotype is more competitive genotype than both Giza 86 and Giza 88 ones. Variation in weed competition strength between the three cotton genotypes may be attributed to their variation in allelopathic potarntial or variation in canopy architecture. Abd El-Bary et al (2010) mentioned that hybridization between Giza 89 as female and Giza. 86 as male

in 1989 collected features as compact growth and the plants were highly resistant to fusurium wilt.

Table 3. Effect of cotton genotypes on dry weight of weeds $\left(\mathrm{g} / \mathrm{m}^{2}\right)$ after 75 and 105 days from sowing (combined data of 2011 and 2012).

\begin{tabular}{|c|c|c|c|c|c|c|c|c|}
\hline \multirow{4}{*}{ Cotton genotypes } & \multicolumn{8}{|c|}{ Days after sowing } \\
\hline & \multicolumn{6}{|c|}{75} & \multicolumn{2}{|c|}{105} \\
\hline & \multicolumn{8}{|c|}{ Dry weight of weeds $\left(\mathrm{g} / \mathrm{m}^{2}\right)$} \\
\hline & $\begin{array}{l}\text { Grasses } \\
\text { weeds }\end{array}$ & $\begin{array}{l}\text { Broadleaf } \\
\text { weeds }\end{array}$ & $\begin{array}{l}\text { perennial } \\
\text { weeds }\end{array}$ & $\begin{array}{l}\text { Total } \\
\text { weeds }\end{array}$ & $\begin{array}{l}\text { Grasses } \\
\text { Weeds }\end{array}$ & $\begin{array}{l}\text { Broadleaf } \\
\text { weeds }\end{array}$ & $\begin{array}{l}\text { perennial } \\
\text { weeds }\end{array}$ & $\begin{array}{l}\text { Total } \\
\text { weeds }\end{array}$ \\
\hline Giza 86 & $51.9 a$ & $136.7 a$ & $90.8 a$ & $279.4 a$ & $150.1 \mathrm{a}$ & $485.1 \mathrm{a}$ & $110.1 a$ & $745.3 a$ \\
\hline Giza 88 & $44.1 b$ & $105.7 b$ & $82.3 b$ & $232.1 b$ & $131.5 b$ & $388.1 b$ & $97.5 b$ & $617.1 \mathrm{~b}$ \\
\hline promising hybrid Giza 89 × 86 & $37.6 \mathrm{c}$ & $93.1 \mathrm{c}$ & $73.8 \mathrm{c}$ & $204.5 c$ & $123.9 \mathrm{c}$ & $352.9 \mathrm{c}$ & $89.7 \mathrm{c}$ & $566.5 c$ \\
\hline
\end{tabular}

Means followed by the same alphabetical letters were not statistically significant according to Duncan, $\mathrm{s}$ multiple range test.

\section{2-Effect of weed control treatments:}

Data presented in Table (4) indicate that different weed control packages depressed the dry matter weight of different weed categories namely grasses, broadleaved, perennial and total weeds than control treatment at 75 and 105 days from sowing. The highest control percentages were obtained from pendimelthalin/hoeing, butralin/hoeing packages and then followed by hand hoeing twice treatment which were $89.96,88.98$ and $82.6 \%$ of total weeds at 75 days after sowing, respectively. These results had the same trend at 105 days from sowing. 
These results are logic because there is no solely weed control treatment is sufficient by it self, and herbicidal hoeing integration is needed for sufficient weed control in cotton due to the longevity of plant season and its poor competition to weeds. Several investigators reported that dinitroaniline as pendimethalin were effective in controlling cotton summer weeds (Fayed et al. 1983 and Khan et al. 1994). In this respect Fayed et al. (1983) reported that applying one supplementary hoeing in cotton herbicidal treatments was necessary to eliminate the weed plants which survived or escaped from the herbicides and to achieve promising weed control along the growing season of cotton plants.

Table 4. Effect of weed control treatments after 75 and 105 days from sowing (combined data of 2011 and 2012).

\begin{tabular}{|c|c|c|c|c|c|c|c|c|}
\hline \multirow{4}{*}{ Weed control packages } & \multicolumn{8}{|c|}{ Days after sowing } \\
\hline & \multicolumn{4}{|c|}{75} & \multicolumn{4}{|c|}{105} \\
\hline & \multicolumn{8}{|c|}{ Dry weight of weeds $\left(\mathrm{g} / \mathrm{m}^{2}\right)$} \\
\hline & $\begin{array}{l}\text { Grasses } \\
\text { weeds }\end{array}$ & $\begin{array}{c}\text { Broadleaf } \\
\text { weeds }\end{array}$ & $\begin{array}{c}\text { perennial } \\
\text { weeds }\end{array}$ & $\begin{array}{l}\text { Total } \\
\text { weeds }\end{array}$ & $\begin{array}{l}\text { Grasses } \\
\text { Weeds }\end{array}$ & $\begin{array}{c}\text { Broadleaf } \\
\text { weeds }\end{array}$ & $\begin{array}{c}\text { perennial } \\
\text { weeds }\end{array}$ & $\begin{array}{l}\text { Total } \\
\text { weeds }\end{array}$ \\
\hline Pendimethalin $(1.7 \mathrm{l} / \mathrm{f}) /$ hand hoeing. & $13.1 \mathrm{~b}$ & $18.2 \mathrm{~d}$ & $34.1 \mathrm{~b}$ & $65.3 \mathrm{~d}$ & $48.8 \mathrm{~b}$ & $130.3 \mathrm{c}$ & $38.4 b c$ & $217.6 \mathrm{~d}$ \\
\hline Butralin $(2.5 \mathrm{l} / \mathrm{f}) /$ hand hoeing & $12.7 \mathrm{~b}$ & $21.8 \mathrm{~cd}$ & $30.6 b$ & $65.2 d$ & $35.9 b$ & $194.0 \mathrm{c}$ & $39.1 \mathrm{bc}$ & $269.0 \mathrm{~cd}$ \\
\hline Fluazifop -p-butyl $(1.5 \mathrm{l} / \mathrm{f}) /$ hand hoeing & $5.5 b$ & $108.7 \mathrm{~b}$ & $22.9 b$ & $137.2 \mathrm{bc}$ & $18.6 \mathrm{~b}$ & $323.3 b$ & $23.0 \mathrm{c}$ & $365.0 \mathrm{bc}$ \\
\hline Sethoxydim $(1.5 \mathrm{l} / \mathrm{f}) /$ hand hoeing & $7.0 \mathrm{~b}$ & $116.8 \mathrm{~b}$ & $26.2 b$ & $150.0 \mathrm{~b}$ & $17.1 \mathrm{~b}$ & $354.1 \mathrm{~b}$ & $42.5 b$ & $413.7 b$ \\
\hline Hand hoeing twice & $15.1 b$ & $49.8 \mathrm{c}$ & $37.8 \mathrm{~b}$ & $102.8 \mathrm{~cd}$ & $23.1 \mathrm{~b}$ & $340.1 b$ & $40.6 \mathrm{bc}$ & $403.9 b$ \\
\hline Control (untreated) & $120.0 a$ & $295.5 a$ & $175.9 a$ & $591.3 a$ & $423.4 a$ & $1006.2 \mathrm{a}$ & $210.8 a$ & $1640.5 a$ \\
\hline
\end{tabular}

Means followed by the same alphabetical letters were not statistically significant according to Duncan, $s$ multiple range test.

\section{3-The effect of interaction between genotypes and weed control treatments:-}

Table (5) show that the effect of interaction of cotton genotypes with weed control packages on dry weight of broad-leaved, grasses, perennial weeds and total weeds $\left(\mathrm{g} / \mathrm{m}^{2}\right)$ at 75 and 105 days from sowing was statistically significant at $5 \%$ level.

Fluazifop -p-butyl + hand hoeing package under promising Giza $89 \times 86$ exerted the highest percentage of controlling of grassy weeds. This result is owing to the sensitivity of grasses to Fluazifop -p-butyl herbicide in addition to the high competition strength of the promising Giza 89x 86 genotype plants against grassy weeds (Table 3). Similar results were obtained by Dilbaugh et al. (2009) whom indicated that pendimelthalin gave $82 \%$ control of broad-leaf weeds and $84.1 \%$ of narrow leaf. 
The highest controlling percentages of broad leaf weeds were obtained from pendimethalin application followed by one hand hoeing which was under studied cotton hybrid genotype as compared with the other genotypes. This might be attributed to the susceptibility of broad-leaf weeds to pendimethalin herbicide integrated with the drastic competitive effect of cotton hybrid on weeds.

The studied interaction was statistically significant on dry weight of perennial weeds at 75 and 105 days from sowing. Flusifop-p-butyl + hoeing packages, under promising G89xG86 exerted the highest controlling percentage of perennial weeds. This result is owing to the role this herbicidal integrated with the role of this cotton genotype.

Concerning the effect of interaction between cotton genotypes under weed control packages on dry weight of total weeds at both 75 and 105 days from sowing, the highest controlling \% was achieved with pendimethalin/ hand hoeing packages under cotton hybrid. This is may be attributed it's the integrated effects of cotton genotype, herbicide and hoeing in maximizing weed control for the aforementioned weed categories in Table (5). 
Table 5. The effect of interaction between cotton genotypes and weed control treatments after 75 and 105 days from sowing in 2011 and 2012 summer seasons.

\begin{tabular}{|c|c|c|c|c|c|c|c|c|c|}
\hline \multirow{4}{*}{ 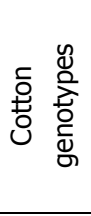 } & \multirow{4}{*}{ Weed control packages } & \multicolumn{8}{|c|}{ Days after sowing } \\
\hline & & \multicolumn{4}{|c|}{75} & \multicolumn{4}{|c|}{105} \\
\hline & & \multicolumn{8}{|c|}{ Dry weight of weeds in $\left(\mathrm{g} / \mathrm{m}^{2}\right)$} \\
\hline & & $\begin{array}{l}\text { Grasses } \\
\text { weeds }\end{array}$ & $\begin{array}{l}\text { Broadleaf } \\
\text { weeds }\end{array}$ & $\begin{array}{l}\text { perennial } \\
\text { weeds }\end{array}$ & $\begin{array}{l}\text { Total } \\
\text { weeds }\end{array}$ & $\begin{array}{l}\text { Grasses } \\
\text { Weeds }\end{array}$ & $\begin{array}{l}\text { Broadleaf } \\
\text { weeds }\end{array}$ & $\begin{array}{l}\text { perennial } \\
\text { weeds }\end{array}$ & $\begin{array}{l}\text { Total } \\
\text { weeds }\end{array}$ \\
\hline $\begin{array}{l}\infty \\
\infty \\
\mathbb{N} \\
\mathbb{N}\end{array}$ & $\begin{array}{l}\text { Pendimethalin }(1.7 \mathrm{l} / \mathrm{f}) / \text { hand hoeing. } \\
\text { Butralin }(2.5 \mathrm{l} / \mathrm{f}) / \text { hand hoeing } \\
\text { Fluazifop -p-butyl (1.5 l/f) / hand } \\
\text { hoeing } \\
\text { Sethoxydim }(1.5 \mathrm{l} / \mathrm{f}) / \text { hand hoeing } \\
\text { Hand hoeing twice } \\
\text { Control (untreated) }\end{array}$ & $\begin{array}{l}22.7 \mathrm{~b} \\
19.7 \mathrm{~b} \\
9.3 \mathrm{~b} \\
13.3 \mathrm{~b} \\
31.8 \mathrm{~b} \\
215.0 \mathrm{a}\end{array}$ & $\begin{array}{l}\text { 36.3ghi } \\
40.7 \mathrm{ghi} \\
137.5 \mathrm{~cd} \\
140.7 \mathrm{~cd} \\
51.7 \mathrm{fghi} \\
413.2 \mathrm{a}\end{array}$ & $\begin{array}{l}63.7 \mathrm{c} \\
46.7 \mathrm{~cd} \\
35.3 \mathrm{de} \\
44.2 \mathrm{~cd} \\
68.2 \mathrm{c} \\
286.5 \mathrm{a} \\
\end{array}$ & $\begin{array}{l}\text { 122.7efgh } \\
\text { 107.0efgh } \\
182.2 \mathrm{de} \\
198.2 \mathrm{~d} \\
151.7 \mathrm{def} \\
914.7 \mathrm{a} \\
\end{array}$ & $\begin{array}{l}88.8 \mathrm{~cd} \\
71.7 \mathrm{~cd} \\
35.0 \mathrm{~d} \\
34.5 \mathrm{~d} \\
42.2 \mathrm{~d} \\
628.3 \mathrm{a}\end{array}$ & $\begin{array}{l}188.3 \mathrm{fghi} \\
272.3 \mathrm{fgh} \\
461.3 \mathrm{cde} \\
518.3 \mathrm{~cd} \\
545.0 \mathrm{c} \\
1525.0 \mathrm{a}\end{array}$ & $\begin{array}{l}69.8 \mathrm{de} \\
71.2 \mathrm{de} \\
45.0 \mathrm{de} \\
73.2 \mathrm{de} \\
70.5 \mathrm{~b} \\
331.3 \mathrm{a}\end{array}$ & $\begin{array}{l}346.9 \mathrm{def} \\
415.2 \mathrm{de} \\
541.3 \mathrm{~cd} \\
626.0 \mathrm{c} \\
657.7 \mathrm{c} \\
2484.7 \mathrm{a}\end{array}$ \\
\hline $\begin{array}{l}\infty \\
\infty \\
\mathbb{N} \\
\mathbb{N}\end{array}$ & $\begin{array}{l}\text { Pendimethalin }(1.7 \mathrm{l} / \mathrm{f}) / \text { hand hoeing. } \\
\text { Butralin }(2.5 \mathrm{l} / \mathrm{f}) / \text { hand hoeing } \\
\text { Fluazifop -p-butyl (1.5 l/f) / hand } \\
\text { hoeing } \\
\text { Sethoxydim }(1.5 \mathrm{l} / \mathrm{f}) / \text { hand hoeing } \\
\text { Hand hoeing twice } \\
\text { Control (untreated) }\end{array}$ & $\begin{array}{l}12.0 \mathrm{~b} \\
12.3 \mathrm{~b} \\
5.2 \mathrm{~b} \\
5.5 \mathrm{~b} \\
10.3 \mathrm{~b} \\
99.3 \mathrm{~b}\end{array}$ & $\begin{array}{l}\text { 16.7hi } \\
20.3 \mathrm{hi} \\
103.2 \mathrm{def} \\
120.50 \mathrm{cde} \\
65.3 \mathrm{efgh} \\
\text { 308.3b } \\
\end{array}$ & $\begin{array}{l}28.3 \mathrm{de} \\
33.5 \mathrm{de} \\
24.5 \mathrm{de} \\
24.7 \mathrm{de} \\
33.0 \mathrm{de} \\
169.5 \mathrm{~b} \\
\end{array}$ & $\begin{array}{l}\text { 57.0ghi } \\
66.2 \mathrm{ghi} \\
132.8 \mathrm{defg} \\
150.7 \mathrm{defg} \\
108.7 \mathrm{efgh} \\
577.2 \mathrm{~b} \\
\end{array}$ & $\begin{array}{l}46.7 \mathrm{~cd} \\
28.7 \mathrm{~d} \\
17.0 \mathrm{~d} \\
13.8 \mathrm{~d} \\
19.7 \mathrm{~d} \\
483.3 \mathrm{~b} \\
\end{array}$ & $\begin{array}{l}\text { 142.0ghi } \\
\text { 192.8fghi } \\
\text { 302.2efghi } \\
\text { 338.5def } \\
\text { 336.2 defg } \\
1017.2 \mathrm{~b} \\
\end{array}$ & $\begin{array}{l}30.8 \mathrm{fg} \\
29.2 \mathrm{fg} \\
13.9 \mathrm{fg} \\
37.8 \mathrm{fg} \\
26.0 \mathrm{fg} \\
207.3 \mathrm{C}\end{array}$ & $\begin{array}{l}219.5 \mathrm{efg} \\
250.7 \mathrm{efg} \\
333.1 \mathrm{ef} \\
390.2 \mathrm{de} \\
381.8 \mathrm{de} \\
1707.8 \mathrm{~b} \\
\end{array}$ \\
\hline 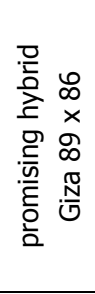 & $\begin{array}{l}\text { Pendimethalin }(1.7 \mathrm{l} / \mathrm{f}) / \text { hand hoeing. } \\
\text { Butralin }(2.5 \mathrm{l} / \mathrm{f}) / \text { hand hoeing } \\
\text { Fluazifop -p-butyl (1.5 l/f) / hand } \\
\text { hoeing } \\
\text { Sethoxydim }(1.5 \mathrm{l} / \mathrm{f}) / \text { hand hoeing } \\
\text { Hand hoeing twice } \\
\text { Control (untreated) }\end{array}$ & $\begin{array}{l}4.7 \mathrm{~b} \\
6.2 \mathrm{~b} \\
2.0 \mathrm{~b} \\
2.2 \mathrm{~b} \\
3.3 \mathrm{~b} \\
45.7 \mathrm{a}\end{array}$ & $\begin{array}{l}1.7 \mathrm{i} \\
4.7 \mathrm{i} \\
85.7 \mathrm{defg} \\
89.3 \mathrm{defg} \\
32.5 \mathrm{ghi} \\
165.0 \mathrm{c}\end{array}$ & $\begin{array}{l}10.2 \mathrm{e} \\
11.7 \mathrm{e} \\
9.0 \mathrm{e} \\
9.7 \mathrm{e} \\
12.3 \mathrm{e} \\
71.7 \mathrm{c}\end{array}$ & $\begin{array}{l}16.5 \mathrm{i} \\
22.5 \mathrm{i} \\
96.7 \mathrm{fgh} \\
101.7 \mathrm{fgh} \\
48.7 \mathrm{hi} \\
282.3 \mathrm{c}\end{array}$ & $\begin{array}{l}11.0 \mathrm{~d} \\
7.3 \mathrm{~d} \\
4.0 \mathrm{~d} \\
2.8 \mathrm{~d} 7 \\
7.3 \mathrm{~d} \\
158.7 \mathrm{c}\end{array}$ & $\begin{array}{l}60.6 \mathrm{i} \\
116.8 \mathrm{hi} \\
206.7 \mathrm{fghi} \\
205.5 \mathrm{fghi} \\
139.5 \mathrm{hi} \\
476.7 \mathrm{cde}\end{array}$ & $\begin{array}{l}14.8 \mathrm{fg} \\
17.2 \mathrm{fg} \\
10.2 \mathrm{~g} \\
16.8 \mathrm{fg} \\
25.6 \mathrm{fg} \\
93.8 \mathrm{~d}\end{array}$ & $\begin{array}{l}86,4 \mathrm{~g} \\
141.3 \mathrm{~g} \\
220.8 \mathrm{efg} \\
225.1 \mathrm{efg} \\
172.4 \mathrm{fg} \\
729.2 \mathrm{c}\end{array}$ \\
\hline
\end{tabular}

Means followed by the same alphabetical letters were not statistically significant according to Duncan,s multiple range test. 


\section{B- Effect on seed cotton yield and its components:-}

Data of ANOVA in Table (6) indicate that the effect of each of cotton genotypes or weed control packages on all studied characters were statistically significantly except lint\%, and the effects of the interaction between genotypes and weed control packages on plant length, boll weight and seed cotton yield were statistically significant where on the rest traits the effect of the interaction were not statistically significant. Also, data in Table (6) show that the effect of interaction between cotton genotypes and weed control packages on plant height $(\mathrm{cm})$, number of fruiting branches per plant, number of bolls/plant and seed index was not statistically significant, meaning that the two studied factors act independent. Meanwhile, the effect of interaction between cotton genotypes and weed control packages on plant height $(\mathrm{cm})$, boll weight $(\mathrm{g})$, seed cotton yield quintar/fad. and lint\% were statistically significant.

Table 6. Means squares of analysis of variance for the effect of cotton genotypes, weed control and their interactions on the growth, yield and yield component (combined data of 2011 and 2012).

\begin{tabular}{|c|c|c|c|c|c|c|c|c|}
\hline Source & DF & $\begin{array}{c}\text { Plant length } \\
\text { (cm) }\end{array}$ & $\begin{array}{l}\text { No. of } \\
\text { fruiting } \\
\text { branches }\end{array}$ & $\begin{array}{l}\text { No. of } \\
\text { bolls } \\
\text { / plant }\end{array}$ & $\begin{array}{l}\text { Boll } \\
\text { weight } \\
\text { (g) }\end{array}$ & $\begin{array}{l}\text { Seed } \\
\text { index }\end{array}$ & $\begin{array}{l}\text { Seed cotton } \\
\text { yield } \\
\text { (quintar } \\
\text { /fad.) }\end{array}$ & Lint $\%$ \\
\hline Year ( y) & 1 & 2.676 & 0.13 & 0.021 & $0.388 * *$ & $47.07 * *$ & $81.085^{* *}$ & $535.113 * *$ \\
\hline Replications x Year & 4 & 87057* & $12.213^{*}$ & 1.252 & $0.080^{*}$ & $2.469 *$ & 0.106 & 6.313 \\
\hline Genotypes ( G ) & 2 & $3220.75 * *$ & $27.82 * *$ & $54.606 * *$ & $1.113 * *$ & $11.713 * *$ & $13.639 * *$ & 9.628 \\
\hline YG & 2 & 113.676 & $11.682^{*}$ & 3.165 & 0.005 & $5.754 * *$ & $1.421^{* *}$ & $12.171^{*}$ \\
\hline Error & 8 & 536.41 & 5.39 & 4.411 & 0.041 & 1.444 & 0.147 & 11.88 \\
\hline Weed control treatments ( $\mathrm{T}$ ) & 5 & $4029.15^{* *}$ & $58.94 * *$ & $65.95 * *$ & $1.884 * *$ & $7.737 * *$ & $54.813^{* *}$ & 14.27 \\
\hline YT & 5 & 77.298 & 1.72 & $3.661^{*}$ & $0.092 *$ & $3.071^{* *}$ & $5.708 * *$ & 10.796 \\
\hline GT & 10 & $248.05^{*}$ & 1.56 & 3.014 & $0.302 * *$ & 0.945 & $1.264 * *$ & 19.446 \\
\hline YGT & 10 & 73.99 & 2.593 & $3.351^{*}$ & 0.015 & $5.173 * *$ & $0.920 * *$ & 10.32 \\
\hline Error & 60 & 217.25 & 4.09 & 3.052 & 0.068 & 1.215 & 0.181 & 10.978 \\
\hline
\end{tabular}

Values followed by $*$ are significantly $(\mathrm{P}=0.05)$.

\section{Effect of cotton genotypes:}

Data recorded in Table (7) show that the promising hybrid Giza 89 x Giza 86 significantly exceeded Giza 88 and Giza 86 genotypes in all growth characters and seed cotton yield ( quintar /fad) and its attributes except, number of bolls/plant. The 
promising hybrid Giza $89 \times$ Giza 86 recorded the highest values of plants height $(\mathrm{cm})$, number of fruiting branches, boll weight / plant $(\mathrm{g})$, seed index, seed cotton yield/quintar followed by Giza 88 as compared with Giza 86 . These results are confirmed results obtained by Abd El- Bary et al (2010), they mentioned that the promising cross Giza $89 x 86$ characterized by high yielding and out yielded Giza 86 by about $10 \%$ in cotton yield.

Table 7. The yield and yield components as affected by cotton genotypes (combined data of 2011 and 2012).

\begin{tabular}{|c|c|c|c|c|c|c|c|}
\hline Cotton genotypes & $\begin{array}{l}\text { Plant length } \\
\qquad(\mathrm{cm})\end{array}$ & $\begin{array}{l}\text { No. of } \\
\text { fruiting } \\
\text { branches }\end{array}$ & $\begin{array}{l}\text { No. of } \\
\text { bolls } \\
\text { / plant }\end{array}$ & $\begin{array}{c}\text { Boll } \\
\text { weight } \\
\text { (g) }\end{array}$ & $\begin{array}{c}\text { Seed } \\
\text { index (g) }\end{array}$ & $\begin{array}{c}\text { Seed } \\
\text { cotton } \\
\text { yield } \\
\text { (quintar } \\
\text { /fad) }\end{array}$ & $\begin{array}{l}\text { Lint } \\
\%\end{array}$ \\
\hline Giza 86 & $147.2 \mathrm{~b}$ & $19.12 \mathrm{ab}$ & $16.4 \mathrm{~b}$ & $2.53 \mathrm{~b}$ & $8.66 \mathrm{~b}$ & $10.1 \mathrm{c}$ & 36.26 \\
\hline Giza 88 & $156.8 \mathrm{ab}$ & $13.82 \mathrm{c}$ & $17.6 a$ & $2.45 \mathrm{~b}$ & $8.90 \mathrm{~b}$ & $10.5 \mathrm{~b}$ & 36.37 \\
\hline promising hybrid Giza $89 \times 86$ & $166.1 \mathrm{a}$ & $19.20 \mathrm{a}$ & $16.1 \mathrm{ab}$ & $2.78 a$ & $9.74 a$ & $10.8 \mathrm{a}$ & 35.43 \\
\hline
\end{tabular}

Means followed by the same alphabetical letters were not statistically significant according to Duncan, $s$ multiple range test.

\section{2-Effect of weed control packages:}

Data in Table (8) indicated that all used weed control packages as well as hand hoeing increased significantly seed cotton yield and its components. Pendimethalin and butralin treatments followed plus by one hand hoeing for each gave the highest values of plant length, number of furiting branches, number of bolls per plant and seed cotton yield per (quintar /fad) in both seasons followed by fluazifop-p-butyl and sethoxydim plus one hand hoeing and hand hoeing twice. These treatments increased seed cotton yield by $66.7,62.4,56.5,46.4$ and $63.8 \%$ respectively, as compared to control treatment. The influence of such treatments on seed cotton yields had the same trend of the abovementioned yield attributes traits. It is worthwhile to mention that, these treatments which gave the highest values of seed cotton yield were also show lowest dry weight of weeds. In this respect Fayed et al. (1983) and Khan et al (1994) recorded that the highest seed cotton yield with application of pendimethalin and attributed such increase to the increases in seed index, boll weight, number of boll/plant and plant heights which are attributed to minimization of weed crop competition for light, space and mineral uptake. Ikram et al. (2012) found that the 
maximum nutrient losses of NPK were found in control and improving growth and NPK uptake of cotton under weed control by herbicides.

Table 8. The yield and yield components as affected by weed control packages (combined data of 2011 and 2012).

\begin{tabular}{|c|c|c|c|c|c|c|c|}
\hline Weed control packages & $\begin{array}{l}\text { Plant } \\
\text { length } \\
(\mathrm{cm})\end{array}$ & $\begin{array}{l}\text { No. of } \\
\text { fruiting } \\
\text { branch } \\
\text { es }\end{array}$ & $\begin{array}{l}\text { No. of } \\
\text { bolls } \\
\text { / plant }\end{array}$ & $\begin{array}{l}\text { Boll } \\
\text { weig } \\
\text { ht } \\
\text { (g) }\end{array}$ & $\begin{array}{c}\text { Seed } \\
\text { Index }(g)\end{array}$ & $\begin{array}{l}\text { Seed } \\
\text { cotton } \\
\text { yield } \\
\text { (quintar } \\
\text { /fad) }\end{array}$ & Lint \% \\
\hline Pendimethalin $(1.7 \mathrm{l} / \mathrm{f}) /$ hand hoeing & $173.7 a$ & $25.6 a$ & $20.6 a$ & $2.9 a$ & $9.9 a$ & $11.5 a$ & $35.2 b$ \\
\hline Butralin (2.5 l/f) / hand hoeing . & 168.2ab & $25.5 a$ & $19.2 b$ & $2.8 a b$ & $9.7 a b$ & $11.2 \mathrm{a}$ & $36.9 a$ \\
\hline Fluazifop -p-butyl (1.5 l/f) /hand hoeing & $160.3 b c$ & 23.6ab & $17.6 \mathrm{c}$ & $2.8 a b$ & $9.2 a b$ & $10.8 b$ & $36.6 a$ \\
\hline Sethoxydim (1.5 l/f) / hand hoeing . & $157.2 \mathrm{~cd}$ & $20.7 b$ & $16.9 c$ & $2.6 b c$ & $9.0 \mathrm{ab}$ & $10.1 \mathrm{c}$ & $36.2 a b$ \\
\hline Hand hoeing twice & $149.1 d$ & $20.5 b$ & $16.5 c$ & $2.5 \mathrm{c}$ & $8.3 c$ & $11.3 a$ & $36.6 a$ \\
\hline Control (untreated) & 131.7e & $15.7 \mathrm{c}$ & $15.2 d$ & $1.9 \mathrm{~d}$ & $8.4 \mathrm{C}$ & $6.9 \mathrm{~d}$ & $34.7 c$ \\
\hline
\end{tabular}

Means followed by the same alphabetical letters were not statistically significant according to Duncan,s multiple range test.

\section{3-The effect of interaction between genotypes and weed control treatments on yield and its components:-}

Data in Table (9) indicate that all interactions between cotton genotypes and weed control treatments were statistically significant on their effects on plant height $(\mathrm{cm})$, boll weight $(\mathrm{g})$ seed index cotton yield quintar/fad, and lint\% increased seed cotton yield and its components except, for plant length and number of fruiting branches. The tallest plants were obtained from the the promising hybrid Giza $89 x$ Giza 86 with pendimethalin (35.8\%) as compared with the shortest plants whom resulted from untreated check.

The interaction between the promising hybrid Giza $89 \times 86$ with pendimethalin plus one hand hoeing gave the highest values for cotton yield (12.6 quintar/fad) while, the lowest values ( 7.0 quintar/fad) was obtained by Giza 86 with control treatment. The increases in such trait is attributed to the highest weed elimination \% under these treatments than unweeded check, and consequently, decreasing weed/crop competition. 
Table 9. The yield and yield components as affected by interaction between cotton genotypes and weed control treatments at harvest (combined data at 2011 and 2012).

\begin{tabular}{|c|c|c|c|c|c|c|}
\hline $\begin{array}{l}\text { Cotton } \\
\text { genotypes }\end{array}$ & Weed control packages & $\begin{array}{c}\text { Plant length } \\
(\mathrm{cm})\end{array}$ & $\begin{array}{l}\text { Boll } \\
\text { weight } \\
\text { (g) }\end{array}$ & $\begin{array}{l}\text { Seed } \\
\text { index } \\
\text { (g) }\end{array}$ & $\begin{array}{l}\text { Seed cotton } \\
\text { yield } \\
\text { (quintar/fad) }\end{array}$ & Lint \% \\
\hline $\begin{array}{l}0 \\
\mathbb{N} \\
\mathbb{N} \\
\mathbb{N}\end{array}$ & 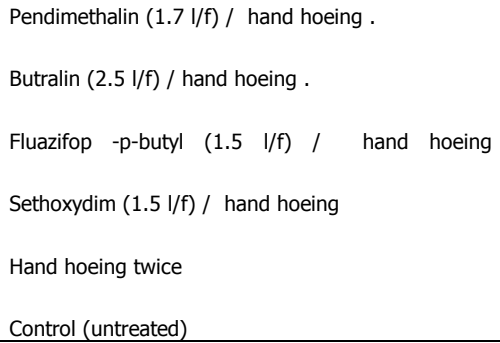 & $\begin{array}{l}153.0 \mathrm{def} \\
155.5 \mathrm{def} \\
154.3 \mathrm{def} \\
151.8 \mathrm{def} \\
146.7 \mathrm{ef} \\
121.8 \mathrm{~g} \\
\end{array}$ & $\begin{array}{l}2.8 \mathrm{a} \\
2.9 \mathrm{a} \\
2.9 \mathrm{a} \\
2.6 \mathrm{abcd} \\
2.36 \mathrm{~cd} \\
1.7 \mathrm{e} \\
\end{array}$ & $\begin{array}{l}9.6 \\
9.3 \\
8.5 \\
8.5 \\
7.9 \\
8.2 \\
\end{array}$ & $\begin{array}{l}11.9 \mathrm{a} \\
11.0 \mathrm{~cd} \\
11.6 \mathrm{ab} \\
11.0 \mathrm{~cd} \\
11.2 \mathrm{bc} \\
7.0\end{array}$ & $\begin{array}{l}37.3 a \\
37.1 a \\
37.0 a \\
36.9 a \\
36.9 a \\
\end{array}$ \\
\hline $\begin{array}{l}\infty \\
\infty \\
\mathbb{N} \\
\mathbb{N}\end{array}$ & 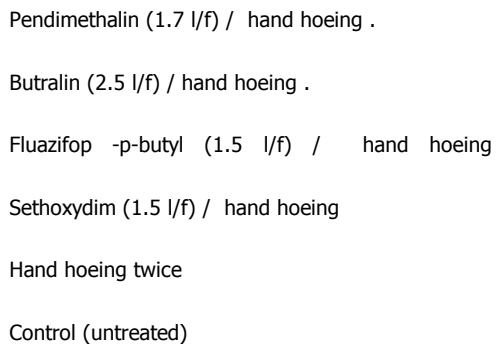 & $\begin{array}{l}178.2 \mathrm{abc} \\
167.2 \mathrm{bcd} \\
155.2 \mathrm{def} \\
157.2 \mathrm{de} \\
146.7 \mathrm{ef} \\
136.3 \mathrm{fg}\end{array}$ & $\begin{array}{l}2.9 \mathrm{abc} \\
2.7 \mathrm{abcd} \\
2.7 \mathrm{abcd} \\
2.4 \mathrm{~d} \\
2.4 \mathrm{bed} \\
1.7 \mathrm{e}\end{array}$ & $\begin{array}{l}9.7 \\
9.5 \\
8.5 \\
8.9 \\
8.2 \\
8.7 \\
\end{array}$ & $\begin{array}{l}11.9 \mathrm{a} \\
11.4 \mathrm{abc} \\
10.9 \mathrm{~cd} \\
10.4 \mathrm{e} \\
11.9 \mathrm{a} \\
7.1 \mathrm{~h}\end{array}$ & $\begin{array}{l}36.8 \mathrm{a} \\
36.3 \mathrm{ab} \\
37.0 \mathrm{a} \\
36.6 \mathrm{a} \\
35.9 \mathrm{ab} \\
35.7 \mathrm{ab}\end{array}$ \\
\hline 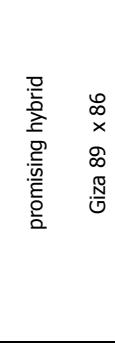 & 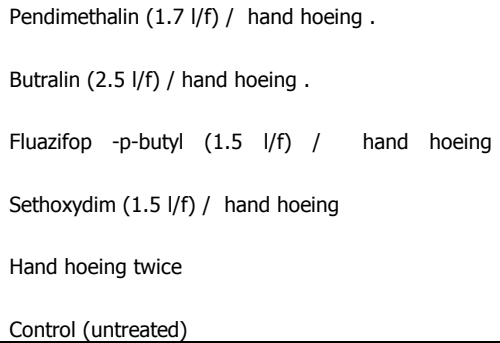 & $\begin{array}{l}189.8 \mathrm{a} \\
182.0 \mathrm{~b} \\
171.5 \mathrm{bcd} \\
162.7 \mathrm{cde} \\
153.8 \mathrm{def} \\
136.8 \mathrm{fg}\end{array}$ & $\begin{array}{l}2.9 a \\
2.8 a \\
2.8 a \\
2.9 a \\
2.7 a b \\
2.6 a b c d\end{array}$ & $\begin{array}{r}10.5 \\
10.5 \\
10.6 \\
9.6 \\
8.9\end{array}$ & $\begin{array}{l}12.6 \mathrm{a} \\
11.1 \mathrm{bcd} \\
9.6 \mathrm{f} \\
8.9 \mathrm{~g} \\
10.9 \mathrm{cde} \\
7.3 \mathrm{i}\end{array}$ & $\begin{array}{c}36.6 a \\
37.1 a \\
35.7 a b \\
34.8 a b c \\
36.9 a\end{array}$ \\
\hline
\end{tabular}

Means followed by the same alphabetical letters were not statistically significant according to Duncan,s multiple range test.

\section{C- Effect on cotton fiber properties:-}

Mean squares of analysis of variance for the effect of cotton genotypes, weed control treatments and their interactions on cotton fiber properties are shown in Table (10). 
Table 10. Means squares of analysis of variance for the effect of cotton genotypes, weed control treatments on cotton fiber properties and their interactions on fiber properties are show (combined data of 2011 and 2012).

\begin{tabular}{|l|c|c|c|c|c|}
\hline Source of variation & DF & $\begin{array}{c}\text { Micronaire } \\
\text { reading }\end{array}$ & $\begin{array}{c}\text { Uniformity } \\
\text { ratio }\end{array}$ & $\begin{array}{c}\text { Pressely } \\
\text { index }\end{array}$ & $\begin{array}{c}\text { Fiber } \\
\text { length }\end{array}$ \\
\hline Year (Y) & 1 & 0.311 & 0.078 & $14.16^{* *}$ & $62.563^{* *}$ \\
\hline RY & 4 & 0.263 & $6.977^{* *}$ & $3.451^{* *}$ & $9.931^{*}$ \\
\hline Genotypes ( G) & 2 & 0.991 & $9.480^{* *}$ & 0.245 & 15.593 \\
\hline YG & 2 & 0.04 & 0.008 & $0.845^{*}$ & 0.023 \\
\hline Error & 8 & 0.347 & 1.387 & 0.749 & 7.172 \\
\hline Treatment ( T) & 5 & $2.963^{* *}$ & 2.095 & 0.535 & 2.844 \\
\hline YT & 5 & 0.011 & 0.009 & 0.747 & 0.014 \\
\hline GT & 10 & 0.189 & 1.273 & 2.087 & 3.691 \\
\hline YGT & 10 & 0.009 & 0.01 & 0.714 & 0.01 \\
\hline Error & 60 & 0.098 & 1,053 & 1.268 & 3.126 \\
\hline
\end{tabular}

Values followed by $*$ are significantly $(p=0.05)$.

\section{1- Effect of cotton genotypes:-}

It is obvious in Table (11) that off the studied four fiber quality measurements, uniformity ratio was significantly affected by genotypes. The promising hybrid gave the lowest value of uniformity ratio whereas the others two genotypes were statistically equal.

Table 11. Fiber quality measurements as affected by cotton genotypes at harvest (combined data of 2011 and 2012 summer seasons).

\begin{tabular}{|l|c|c|c|c|}
\hline Cotton genotypes & $\begin{array}{c}\text { Micronaire } \\
\text { reading }\end{array}$ & $\begin{array}{c}\text { Uniformity } \\
\text { ratio }\end{array}$ & $\begin{array}{c}\text { Pressely } \\
\text { index }\end{array}$ & $\begin{array}{c}\text { Fiber } \\
\text { length }(\mathrm{mm})\end{array}$ \\
\hline Giza 86 & 3.96 & 87.12 & 10.36 & 34.13 \\
Giza 88 & 3.80 & 87.28 & 10.26 & 35.24 \\
Promising hybrid Giza 89 886 & 4.19 & 86.32 & 10.19 & 34.08 \\
\hline
\end{tabular}

Means followed by the same alphabetical letters were not statistically significant according to Duncan,s multiple range test.

\section{2- Effect of weed control packages:-}

Data in Table (12) indicated that micronaire reading increased significantly under various weed control packages namely pendimethalin/hoeing, butralin /hoeing, fluazifop/hoeing, sethoxydim/hoeing and hand hoeing twice by 33.9, 34.5, 30.5 and 
$25.16 \%$ than untreated check respectively. The increases in micronaire reading of cotton obtained from weed control packages may be attributed to successful control weeds which reduced competition and consequently favored growth, cotton yield and lint maturity. These results are in harmony with those obtained by Keeley and Thullen (1975) whom mentioned that the micronaire reading were lower for cotton sample collected from plots contained nutsedge than weed free plots indicating delayed crop and fiber maturity. Nabil et al. (1983) and El-Shaer et al. (1983) stated that the herbicide stomp gave the highest micronaire $r$ reading, fiber length and fiber strength.

Table 12. The combined data for cotton fiber properties as affected by weed control treatments in 2011 and 2012 seasons.

\begin{tabular}{|l|c|c|c|c|}
\hline \multicolumn{1}{|c|}{ Weed control packages } & $\begin{array}{c}\text { Micronaire } \\
\text { reading }\end{array}$ & $\begin{array}{c}\text { Uniformity } \\
\text { ratio }\end{array}$ & $\begin{array}{c}\text { Pressely } \\
\text { index }\end{array}$ & $\begin{array}{c}\text { Fiber } \\
\text { length (mm) }\end{array}$ \\
\hline Pendimethalin (1.7 l/f) / hand hoeing . & & & & \\
Butralin (2.5 l/f) / hand hoeing . & $4.26 \mathrm{a}$ & 86.93 & 10.38 & 34.51 \\
Fluazifop -p-butyl (1.5 l/f) / hand & $4.26 \mathrm{a}$ & 86.92 & 10.09 & 35.13 \\
hoeing Sethoxydim (1.5 l/f) / hand & $4.15 \mathrm{ab}$ & 87.01 & 10.27 & 34.07 \\
hoeing & $4.07 \mathrm{ab}$ & 87.23 & 10.08 & 34.66 \\
Hand hoeing twice & $3.98 \mathrm{~b}$ & 86.25 & 10.53 & 34.48 \\
Control (untreated) & $3.18 \mathrm{c}$ & 87.1 & 10.28 & 34.07 \\
\hline
\end{tabular}

Means followed by the same alphabetical letters were not statistically significant according to Duncan,s multiple range test.

\section{3-The effect of interaction between genotypes and weed control treatments:}

The effect of interaction between cotton genotypes and weed control treatments on cotton fiber micronaire reading was statistically significant (Table 13). The highest micronaire reading was obtained with promising hybrid with pendimthalin application (4.50) while the lowest value were obtained from Giza 86 under untreated check (3.05). These results mean that cotton fiber maturity can be enhanced with weed elimination from cotton fields. Similar results were obtained by Keeley and Thullen (1975) whom mentioned that the micronaire reading were lower for cotton sample collected from plots contained nutsedge than weed free plots indicating delayed crop and fiber maturity. 
Table 13. The micronaire reading as affected by interaction between cotton genotypes and weed control treatments as quality measurement of cotton genotypes.

\begin{tabular}{|c|c|c|c|}
\hline \multirow[b]{2}{*}{ Weed control packages } & \multicolumn{3}{|c|}{ Cotton genotype } \\
\hline & Giza 86 & Giza 88 & $\begin{array}{c}\text { promising } \\
\text { hybrid } \\
\text { Giza } 89 \times 86\end{array}$ \\
\hline Pendimethalin (1.7 l/f) / hand hoeing . & $4.17 \mathrm{~b}$ & $4.10 \mathrm{a}$ & $4.50 a$ \\
\hline Butralin $(2.5 \mathrm{I} / \mathrm{f}) /$ hand hoeing . & 4.45ab & $4.00 \mathrm{ab}$ & 4.32ba \\
\hline Fluazifop -p-butyl $(1.5 \mathrm{l} / \mathrm{f}) /$ hand hoeing & $3.93 b$ & 4.07ab & $4.45 a$ \\
\hline Sethoxydim (1.5 l/f) / hand hoeing & 4.05ab & $3.85 a b$ & $4.30 \mathrm{ab}$ \\
\hline Hand hoeing twice & $4.10 a b$ & $3.67 \mathrm{~b}$ & $4.17 b$ \\
\hline Control (untreated) & $3.05 c$ & $3.13 c$ & $3.37 c$ \\
\hline
\end{tabular}

Means followed by the same alphabetical letters were not statistically significant according to Duncan, $\mathrm{s}$ multiple range test.

\section{Correlation between studied characters and cotton yield:}

Data presented in Table (14) indicated clearly that simple correlation coefficients between dry weight of grassy weeds and broad-leaved weeds species and cotton yield was statistically significant and negative at $5 \%$ level. Such correlation was strong with broad-leaved weeds $(-0.946,-0.774$ and -0.903$)$ than with grassy weeds $(-0.865,-0.756$ and -0.551$)$ for the three genotype of cotton (Giza 86 , Giza 88 and promising hybrid Giza $89 \times 86$ ), respectively. This mean that broad-leaved were more aggressive in their competition to cotton genotypes than grassy weeds. Correlation between dry weight of total annual weeds and seed cotton yield recorded the highest value, where negatively affected cotton yield by $(-0.960,-0.783$ and -0.876$)$ for the three genotypes of cotton (Giza 86, Giza 88 and promising hybrid Giza $89 \times 86$ ), respectively. 
Table 14. Correlation coefficient between studied characters and some cotton genotypes yield, (combined data of 2011 and 2012).

\begin{tabular}{|c|c|c|c|c|c|c|c|c|}
\hline 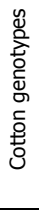 & Studied characters & $\begin{array}{l}\text { Grassy } \\
\text { weeds } \\
\left(\mathrm{g} / \mathrm{m}^{2}\right)\end{array}$ & $\begin{array}{c}\text { Total } \\
\text { weeds }(\mathrm{g} / \\
\left.\mathrm{m}^{2}\right)\end{array}$ & $\begin{array}{l}\text { Plant height } \\
\text { (cm) }\end{array}$ & $\begin{array}{c}\text { Boll } \\
\text { weight/ } \\
\text { (g) }\end{array}$ & $\begin{array}{c}\text { Pressely } \\
\text { index }\end{array}$ & $\begin{array}{l}\text { Fiber } \\
\text { length }\end{array}$ & $\begin{array}{l}\text { Seed cotton } \\
\text { yield } \\
\text { (quintar } \\
\text { /fad) }\end{array}$ \\
\hline \multirow{7}{*}{$\begin{array}{l}\infty \\
\infty \\
\mathbb{N} \\
\stackrel{N}{0}\end{array}$} & Broad-leaved weeds $(\mathrm{g} / \mathrm{m} 2)$ & $0.853 * *$ & $0.987 * *$ & $-0.656 * *$ & $-0.853 * *$ & -0.223 & -0.283 & $-0.946 * *$ \\
\hline & Grassy weeds (g/m2) & - & $0.915 * *$ & -0.376 & $-0.782 * *$ & -0.044 & -0.383 & $-0.865 * *$ \\
\hline & Total weeds $(\mathrm{g} / \mathrm{m} 2)$ & & - & $-0.593 *$ & $-0.862 * *$ & 0.204 & -0.295 & $-0.960 * *$ \\
\hline & Plant height $(\mathrm{cm})$ & & & - & $0.504 *$ & 0.324 & -0.158 & $0.576 *$ \\
\hline & Boll weight $/(\mathrm{g})$ & & & & - & -0.464 & -0.062 & $0.703 * *$ \\
\hline & Pressely index & & & & & - & -0.227 & -0.172 \\
\hline & Fiber length & & & & & & - & 0.238 \\
\hline \multirow{7}{*}{$\begin{array}{l}\infty \\
\infty \\
\mathbb{N} \\
\mathbb{N} \\
\mathbb{N}\end{array}$} & Broad-leaved weeds $(\mathrm{g} / \mathrm{m} 2)$ & $0.914 * *$ & $0.989 * *$ & -0.397 & $-0.764 * *$ & -0.138 & -0.351 & $-0.774 * *$ \\
\hline & Grassy weeds $(\mathrm{g} / \mathrm{m} 2)$ & - & $0.962 * *$ & -0.430 & $-0.616 * *$ & -0.048 & -0.306 & $-0.756 * *$ \\
\hline & Total weeds $(\mathrm{g} / \mathrm{m} 2)$ & & - & -0.413 & $-0.731 * *$ & 0.073 & -0.328 & $-0.783 * *$ \\
\hline & Plant height $(\mathrm{cm})$ & & & - & -0.456 & 0.155 & -0.140 & 0.363 \\
\hline & Boll weight /(g) & & & & - & -0.064 & -0.047 & $0.612 * *$ \\
\hline & Pressely index & & & & & - & -0.096 & -0.112 \\
\hline & Fiber length & & & & & & - & 0.235 \\
\hline \multirow{7}{*}{ 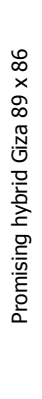 } & Broad-leaved weeds ( $\mathrm{g} / \mathrm{m} 2$ ) & $0.659 * *$ & $0.957 * *$ & $-0.540 *$ & -0.221 & -0.091 & -0.040 & $-0.903 * *$ \\
\hline & Grassy weeds ( $\mathrm{g} / \mathrm{m} 2)$ & - & $0.842 * *$ & -0.211 & $-0.499 *$ & -0.088 & -0.003 & $-0.551 *$ \\
\hline & Total weeds $(\mathrm{g} / \mathrm{m} 2)$ & & - & $-0.474 *$ & $-0.616 * *$ & 0.006 & -0.026 & $-0.876 * *$ \\
\hline & Plant height $(\mathrm{cm})$ & & & - & $0.531 *$ & 0.064 & -0.109 & $0.614 * *$ \\
\hline & Boll weight /(g) & & & & - & -0.076 & 0.120 & $0.732 * *$ \\
\hline & Pressely index & & & & & - & -0.333 & -0.084 \\
\hline & Fiber length & & & & & & - & 0.076 \\
\hline
\end{tabular}

Also, correlation analysis revealed that the yield increases due to type of weed competition were positively contributed to the increases in growth characters and yield components. The correlation between total weeds and cotton yield in three cotton genotypes was highly statistically significant. Hence, applying weed control packages play a major role in increasing cotton productivity and improve fiber maturity per unit urea, when applied at the suitable time, rate and stage of weed growth. 


\section{REFERENCES}

2. Abd El-Bary, A. M. R., H. M. E. Hadmoud, W. M. B. Yehia, M.S.M. Sour, Samia E.S. Ali and Eman M. R. M. Saleh. 2010. The long staple Egyptian cotton promising cross Giza 89xGiza 86. J. Agric. Chemistry and Biotechnology. Mansoura Univ., Vol. (6): 325-339.

3. Abd El-Rehim, Salwa A., Samia A. Saad EL-Din and A. H. A. Afifi. 1995. Comparative study on the effect of hand hoeing and different herbicidal treatments on physical properties of fiber and yarn growth and yield in Egyptian cotton. J. Agric. Sci. Mansoura Univ., 21 (7): 2473-2483.

4. Anomymous. 2012. Brief of Agricultural Statistics, Arab Republic of Egypt, Ministry of Agricultural and Land Reclamation, bulletin, P 30.

5. ASTM. 1984. American Society for Testing and Materials. Standards on textile materials. D: 1447-83, D: 1448-74 and D:1445-75.

6. Buchanan, G. A. and E.R. Burns. 1971. Weed competitions in cotton. Weed Sci, 19: 580-582. 11.

7. Bukun, B., 2004. Critical periods for weed control in cotton in Turkey. Weed Res. 44, 404- 412.

8. Dilbaugh, M., N. A. Muhammad, I. Raza and M. A. Mian. 2009. Growth and development of cotton (Gossypium hirsutum I.) as affected by different methods of pendimethalin application, Pak. J. Weed Sci. Res. 15(1): 11-17,

9. Duncan, B.O. 1955. Multiple range and multiple range $F$ test. Biometrics, 11: 142.

10. El- Shaer, M. H., M. S. Abd El- Raouf, A. M. Samar and H. A. Ali. 1985. Effect of plant population density and weed control treatments on weed dry weight and cotton yield and quality. Annals of Agric. Sci. Ain. Shams Univ., 28 (3): 12911307.

11. Fayed, M.T, M.T. Mostafa and E.E. Hassanein. 1983. Increasing the efficiency of herbicides in controlling cotton weeds by one light hoeing .Proc. First Conf. of Agron. Egyptian Soc. of Crop Sci., 2: 679-688.

12. Ferrel, J.A., G.E. Mac Donald and B. J. Brecke. 2001. Weed management in cotton. University of Florida IFSA Extension, pp, 1-32.(C.F. Asian j. of plant sci. 6(2): 344-348,

13. Ghourab, M.H.H. 1990. Effect of some herbicides on Egyptian cotton. Ph.D. Thesis, Fac. Agri., Al-Azhar Univ., Egypt.

14. Ikram R. M., M. A. Nadeem, A. Tanveer, M. Yasin, A. U. Mohsin, R. N. Abbas, H. Rehman, M. Sibtain and M. Irfan. 2012. Comparative efficacy of different pre- 
emergence herbicides in controlling weeds in cotton (Gossypium hirsutum I.) Pak. J. Weed Sci. Res. 18(2): 209-222.

15. Keeley, P.E., C.H.Carter and J.H. Miller. 1973. Yellow nutsedge and cotton response to several herbicides. Weed Sci. 21:327-329.

16. Keeley, P.E., and R.J. Thullen. 1975. Influence of yellow nutsedge competition on furrow-irrigated cotton. Weed Sci. 23:171-175.

17. Khan, N.U., S.U. Khan, G. Hassan, I. H. Shah and Q. Nawaz. 2001. Studies on weed control in cotton (Gossypium hirsutum L) online, J. Bid. Sci. 1 (3): 143-145.

18. Nabil, Z.S., A. M. Samara and S. H. Yousef. 1983. Effect of different pesticide treatments on fiber and seed characteristics. (Proc. of the first Conf. of Agron., 96: 699-707).

19. Snedecor, G. W. and W. G. Cochran. 1980. " Statistical Methods "6 ${ }^{\text {th }}$ ed., Iowa State Univ. Press, Ames. Iowa, U.S.A, 325-330.

20. Steel, R. G. D. and Jour. H. Torrie. 1980. Principles and Procedures of Statistics. MC. Graw Hill Book Company Inc. New York, 481 pp.

21. Sundaram, V. 1980. Hand book of Methods of Tests for Cotton Fibers, Yarn and Fabrics. Indian Council of Agric. Res., P. 71-76. 


\title{
تأثير بعض حزم المكافحة المتكاملة للحشائش على نمو ومحصول القطن وصفات التيلة لبعض التراكيب الوراثية للقطن والحشائش المصاحبة ومائة
}

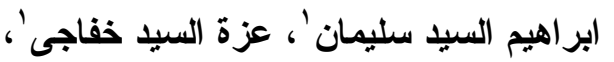

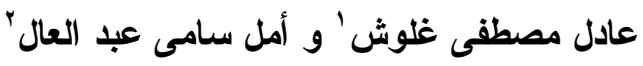 \\ | ا ـ الدعل المركزى لبحوث الحثائش - مركز البحوث الزراعية - الجيزة- مصر

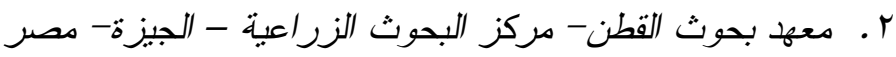

•أجريت تجربتان حقليتان فى مزرعة محطة البحوث الزر اعية بسخا - كفر الثيخ خلال

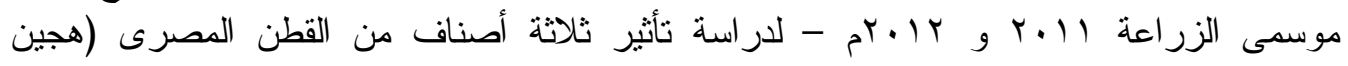

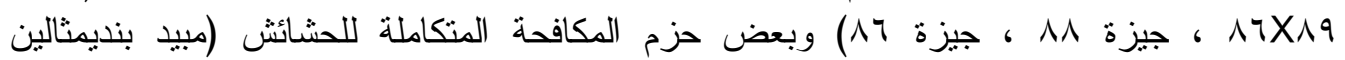

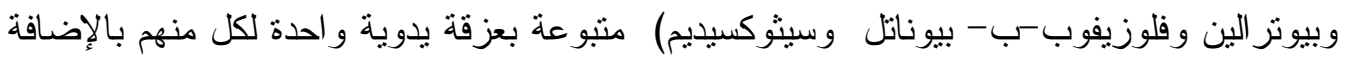

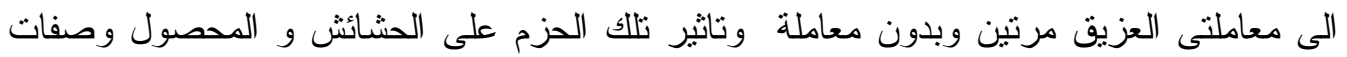
التيلة للقطن.

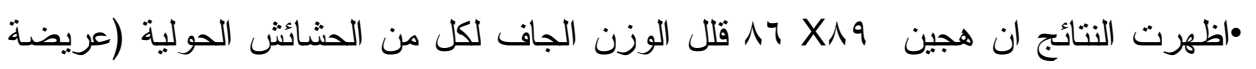

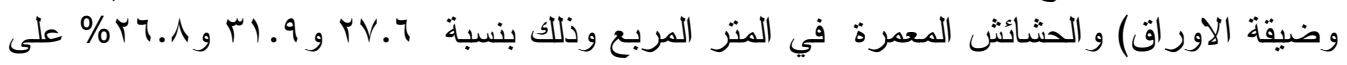

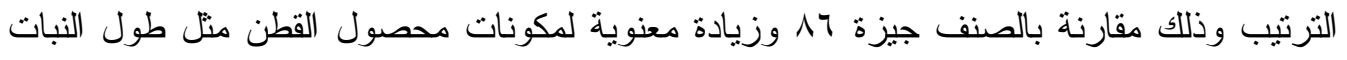

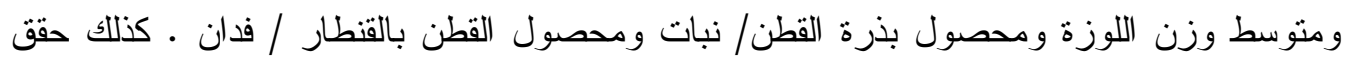

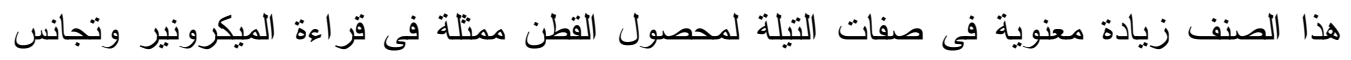

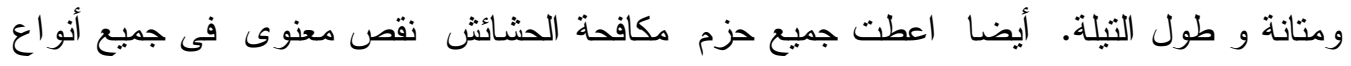

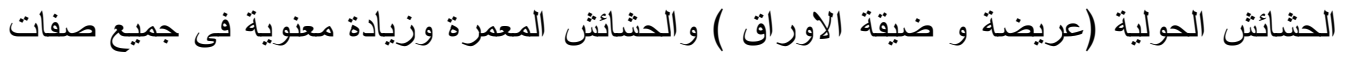

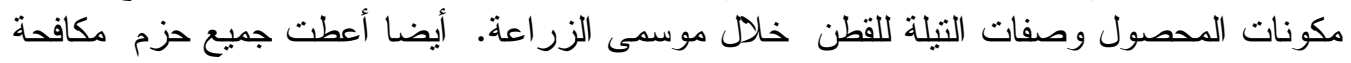

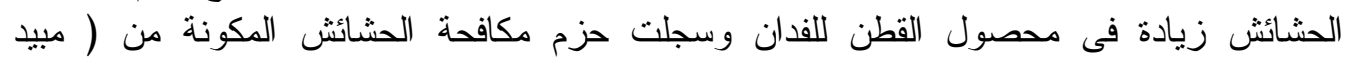

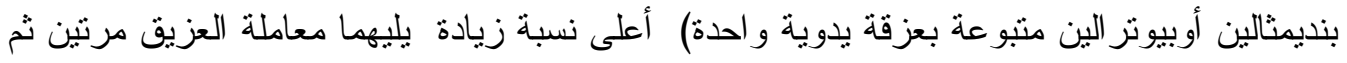

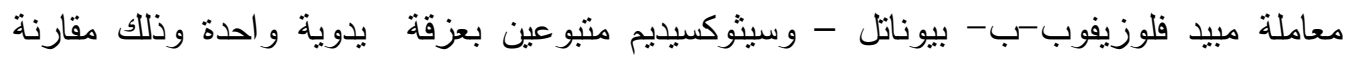

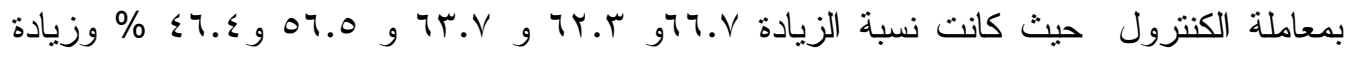

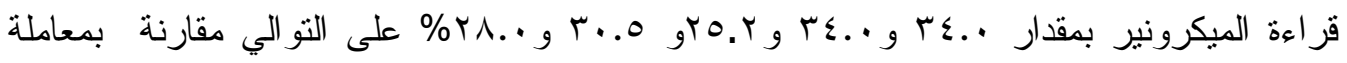

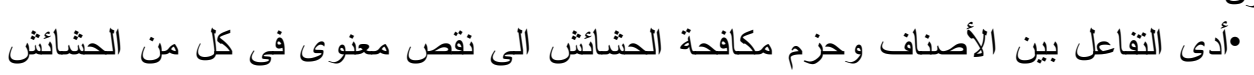

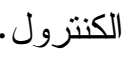

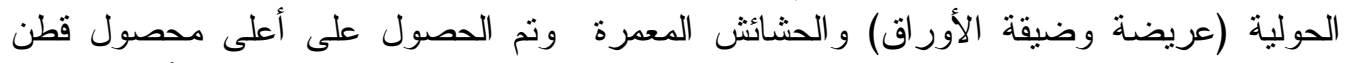

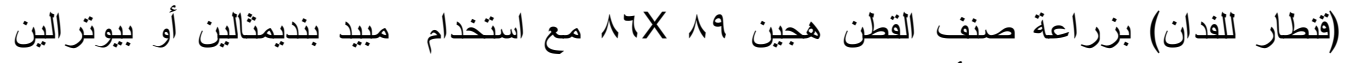

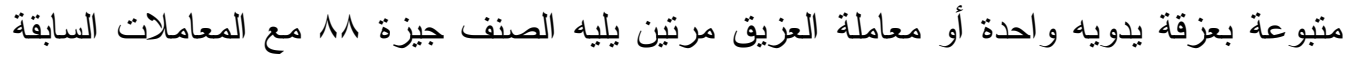

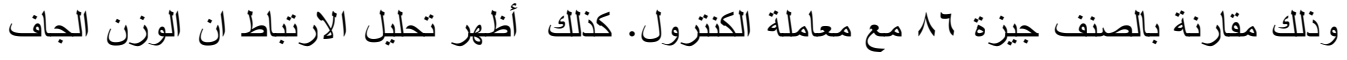

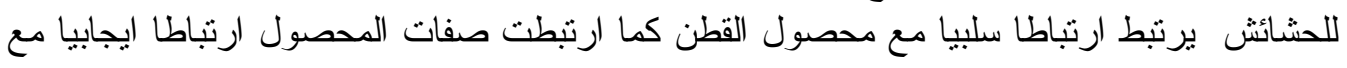

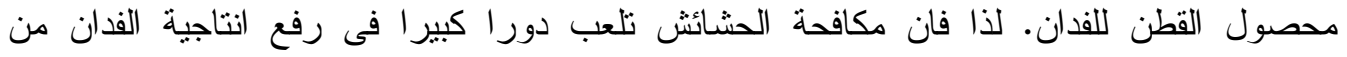
محصول القطن.

• توصى هذه الدراسة باستخدام أصناف القطن المصرى الآكثر تأثير ا على الحشائش مثل

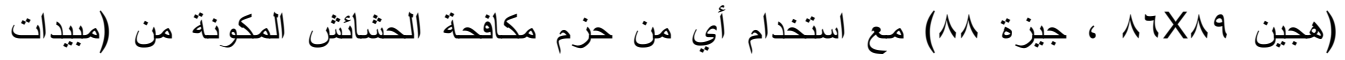

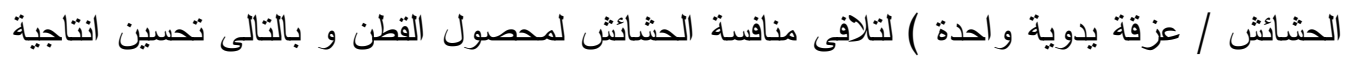

